\title{
Title: The evolution of bacterial shape complexity by a curvature-inducing module
}

Authors: Nicholas R. Martin, Edith Blackman, Benjamin P. Bratton, Thomas M. Bartlett†, Zemer Gitai*.

\section{Affiliations:}

Department of Molecular Biology, Princeton University, Princeton, NJ 08544, USA

*Correspondence to: zgitai@ princeton.edu

†Present address: Department of Microbiology, Harvard Medical School, Boston, MA 02115

\begin{abstract}
Bacteria can achieve a staggering diversity of cell shapes that promote critical functions like growth, motility, and virulence ${ }^{1-4}$. Previous studies suggested that bacteria establish complex shapes by co-opting the core machineries essential for elongation and division $^{5,6}$. In contrast, we discovered a two-protein module, CrvAB, that can curve bacteria autonomously of the major elongation and division machinery by forming a dynamic, asymmetrically-localized structure in the periplasm. CrvAB is essential for curvature in its native species, Vibrio cholerae, and is sufficient to curve multiple heterologous species spanning 2.5 billion years of evolution. Thus, modular shape determinants can promote the evolution of morphological complexity independently of existing cell shape regulation.
\end{abstract}

\section{Main Text:}

Cells come in a variety of shapes that contribute to fitness in diverse environments ${ }^{1,2,7,8}$. In bacteria, cell curvature can influence motility and host colonization ${ }^{1,3,4}$. While bacteria can achieve a huge diversity of shapes, all of these shapes are defined using a rigid cell wall made from a meshwork of peptide-crosslinked polysaccharide strands, termed peptidoglycan $(\mathrm{PG})^{9}$. 
The mechanisms by which bacteria achieve complex shapes remain largely mysterious, but in all cases studied to date they involve species-specific co-opting of the machinery required for making simple shapes ${ }^{5,6}$. For example, MreB, a cytoskeletal organizer required for the formation of simple rod shapes in most bacterial species ${ }^{10,11}$, is required for the elaboration of both curvature and stalk formation in $C$. crescentus $^{5,6}$. In addition to MreB, the other widelyconserved cytoskeletal organizer is FtsZ ${ }^{12,13}$. Each of these cytoskeletal elements localizes asymmetrically to direct the activity of an associated set of PG synthesis enzymes. Here we describe a cell curvature module that can function in multiple species independently of both the MreB- and FtsZ-associated "core" shape machineries, demonstrating that the evolution of bacterial shape is more plastic than previously appreciated.

\section{Two genes, $\operatorname{crv} A$ and $\operatorname{crv} B$, are sufficient to induce cell shape complexity}

To understand the origin of a complex curved-rod shape, we focused on the human pathogen Vibrio cholerae. The only known factor required for cell curvature in V. cholerae, CrvA, forms a protein filament that localizes to the inner face of the cell to induce cell curvature ${ }^{14}$. The

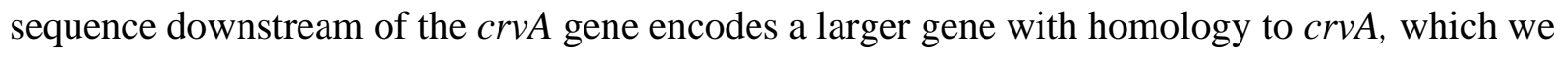
named $\operatorname{crvB}$ (Fig. S1A, B). To determine whether this second gene is also a curvature determinant, we deleted it and found that the resulting cells were straight (Fig. 1A). Complementation tests showed that $\operatorname{crv} A$ complemented $\triangle \operatorname{crv} A$ and $\operatorname{crv} B$ complemented $\Delta \operatorname{crv} B$, but $\operatorname{crvA}$ and $\operatorname{crv} B$ could not cross-complement (Fig. S1C, D, E). We searched for CrvB homologs in the genomes of other Vibrio species and found that almost every Vibrio with a CrvA homolog also has a CrvB homolog (Fig. 1B). The singular exception, Vibrio proteolyticus, contains only a CrvA homolog and is reported to be a straight rod ${ }^{15}$. Furthermore, many species 
have neither a CrvA nor a CrvB homolog and are reported to be straight. Thus, not only are CrvA and CrvB both required for cell curvature in V. cholerae, but their evolutionary cooccurrence correlates with cell curvature throughout the Vibrio genus.

To determine whether CrvA and CrvB are sufficient to induce curvature, we introduced them into the straight-rod species Escherichia coli and Pseudomonas aeruginosa. Despite the fact that $\operatorname{crvA} / \operatorname{crvB}$ homologs are restricted to the family Vibrionaceae, heterologous expression was sufficient to transform both $E$. coli and $P$. aeruginosa into curved rods. Thus, $\operatorname{crv} A$ and $\operatorname{crv} B$ represent the complete set of $V$. cholerae-specific genes required for curvature (Fig. 1C, Fig. S2). We next sought to determine if CrvA and CrvB are sufficient to induce curvature in a species that is not normally a straight rod. V. cholerae, E. coli, and P. aeruginosa are $\gamma$-proteobacteria, while the curved-rod Caulobacter crescentus is an $\alpha$-proteobacterium. C. crescentus lacks $\operatorname{crv} A / \operatorname{crv} B$ homologs but requires crescentin (creS) for curvature such that a $\triangle \operatorname{cre} S$ mutant is a straight rod. CreS does not bear substantial primary sequence homology to CrvA or CrvB ${ }^{14}$. Nevertheless, heterologous CrvA and CrvB expression curved $\triangle$ creS cells (Fig. 1C, Fig S2) and significantly enhanced cell curvature in wild-type C. crescentus (Fig. 1C, Fig. S2). Thus, CrvA and CrvB can function in C. crescentus independently of CreS.

\section{CrvA and CrvB form a module that functions autonomously of core shape machineries}

While V. cholerae, E. coli, P. aeruginosa, and C. crescentus use the MreB-directed machinery, known as the elongasome $5,10,16,17$, to insert new PG throughout the lateral surface of the cell, some bacteria form rods via a distinct growth pattern. For example, the $\alpha$-proteobacterium Agrobacterium tumefaciens lacks many canonical elongasome members including MreB, and 
instead co-opts FtsZ-directed synthesis to elongate from the cell pole ${ }^{18,19}$. Surprisingly, CrvA and CrvB expression induced cell curvature in A. tumefaciens despite its distinct spatial pattern of growth and the absence of MreB (Fig. 1C, Fig. S2). Because CrvA and CrvB are sufficient to induce curvature in multiple heterologous species, including both straight and curved species, as well as in species that use either MreB-driven lateral growth or FtsZ-mediated polar growth, we refer to them as the CrvAB cell shape module.

$V$. cholerae curvature is robust to inhibition of cell division ${ }^{14}$ and the MreB-independent function of the CrvAB module in A. tumefaciens suggests that the elongasome is not required for induction of cell curvature. To further test the dependence of CrvAB on the core shape machineries, we inhibited the elongasome and the FtsZ-associated "divisome" machinery in $E$. coli expressing CrvAB. Treatment with the MreB-disruptor, A22, induced a dose-dependent widening of cells (Fig 1D, Fig. S3A) indicative of disrupted MreB function ${ }^{20}$. Nonetheless, CrvAB expression increased cell curvature over the duration of A22 treatment at all concentrations tested (Fig. 1D, Fig. S3B). CrvAB expression also induced curvature during treatment with the divisome inhibitor, cephalexin, alone or in combination with A22 (Fig. 1E). The ability of the CrvAB module to function independently of both core machines demonstrates that the emergence of complex cell shape can be achieved by the horizontal transfer of only two genes that act autonomously of endogenous cell shape regulation.

\section{The evolution of the CrvAB cell shape module}

CrvA and CrvB share sequence homology, but their inability to complement one another suggests that they may have distinct functions. We thus looked for differences in their amino 
acid sequences and found that CrvB contains a $\sim 250$ amino acid C-terminal domain that is not present in CrvA. Deletion of either this $\underline{\mathrm{C}} \underline{\mathrm{B}} \underline{\mathrm{B}}$ - $\underline{\text { specific }}$ (CBS) domain or the $\underline{\mathrm{N}}$-terminal domain (NTD) of CrvB abolished curvature (Fig S4A). Thus, the CBS domain is necessary, but not sufficient for CrvB function. Remarkably, when we fused the CBS domain to the C-terminus of CrvA, the resulting chimeric protein, CrvA $A_{C B S}$, induced curvature in a $\triangle c r v A B$ background (Fig. 2A, Fig S4A). In V. cholerae, cell curvature rapidly decreases when saturated cultures are diluted into fresh media and increases until the culture reaches stationary phase ${ }^{14}$. While $\operatorname{crv} A_{C B S}$ was sufficient to curve cells, these cells did not become as curved as wild type (Fig 2B). To determine if increasing the level of $c r v A_{C B S}$ could further increase curvature, we expressed a second copy of $c r v A_{C B S}$ at the $c r v B$ locus $\left(2 x C r v A_{C B S}\right)$ and found that this strain reached nearly wild-type curvature (Fig. 2B). However, $\mathrm{CrvA}_{\mathrm{CBS}}$ induced curvature slower than wild type even when two copies were present (Fig. 2B). Therefore, a CrvA/CrvB hybrid functions as a minimal CrvAB module but does so slower than the two separate CrvA and CrvB determinants found in wild type.

The division of functional domains among CrvA and CrvB suggested the possibility that the CrvAB module evolved from an ancestral protein resembling CrvA $\mathrm{CBS}$. We performed a phylogenetic analysis of all sequenced CrvA/CrvB homologs and found a set of sequences that resemble a CrvA $A_{\text {CBS }}$ chimera in clades related to Vibrios such as Allivibrios (Figs. S4B-D). These proteins, which we refer to as CrvY, are distinct from both CrvA and CrvB, as they contain a CBS domain similar to that of CrvB but are more similar to CrvA in the rest of the protein (Fig 2C). Further phylogenetic analysis suggested that the common ancestor of species with CrvAB homologs had a single Crv protein that contained a CBS domain (Figs. S4B-D). To analyze the 
function of CrvY we focused on the curved species Aliivibrio fischeri. Deletion of crvY (VF_A0834) resulted in straight $A$. fischeri cells and heterologous expression of $c r v Y$ in $V$. cholerae was sufficient to induce curvature in a $\triangle c r v A B$ mutant (Fig. 2D, Figs. S4E,F). The necessity of CrvY for curvature in A. fischeri and sufficiency for curvature in $V$. cholerae indicate that $\mathrm{CrvY}$ is a functional, extant form of a CrvA $\mathrm{CBBS}_{\mathrm{C}}$ chimera. Our data thus suggest that the CrvAB module evolved by the duplication of a single ancestral gene followed by the loss of the CrvA CBS domain and divergence of the CrvB N-terminal domain.

\section{CrvAB forms a periplasmic filament that generates asymmetry and governs cell curvature}

\section{dynamics}

How do CrvAB induce an asymmetric cell shape without co-opting the core shape machinery? CrvA tagged with the fluorescent protein msfGFP (CrvA-GFP) is functional and assembles into a filamentous structure localized to the inner curvature of the cell ${ }^{14}$. We found that a CrvBmsfGFP fusion was also functional and formed a filament at the inner curve (Fig. 3A, Fig. S5A). An msfGFP fusion to CrvA $A_{C B S}$ was functional and also localized in the same pattern (Figs. S4A, S5B). When we imaged mCherry-tagged CrvA (CrvA-mCherry) and CrvB-GFP simultaneously, both proteins localized to the same structure (Fig. 3A, Fig. S5A) and remained colocalized as cells grew (Fig. 3B, Movies S1-S2). Furthermore, CrvA-mCherry and CrvB-GFP colocalized upon heterologous expression in E. coli, indicating that CrvAB form an asymmetric structure in a species-independent manner (Fig. 3A, Fig. S5C). Thus, CrvA and CrvB are together sufficient to generate an asymmetric structure in $E$. coli, which could explain why they do not need MreB or FtsZ to break symmetry. 
In Gram-negative bacteria, the cell wall is located between the inner and outer cell membranes in a subcellular compartment called the periplasm. In contrast to cytoskeletal elements, which assemble in the cytoplasm, CrvA is a periskeletal element that assembles in the periplasm ${ }^{14}$. To determine whether CrvB is also periplasmic, we labelled the periplasm by fusing mCherry to the secretion signal of the periplasmic protein DsbA ${ }^{21}$. When we perturbed these cells with the antibiotic moenomycin, cells rounded up and the periplasm visibly protruded from the cytoplasm ${ }^{22}$. As expected for a periplasmic protein, CrvA-GFP filaments localized to the DsbA-mCherrycontaining periplasm ${ }^{14}$ (Fig. 3C). CrvB-GFP filaments also colocalized with DsbA-mCherry, indicating that CrvB is periplasmic. Furthermore, CrvA-mCherry and CrvB-GFP filaments remained colocalized in rounded moenomycin-treated cells, indicating that their colocalization to this periskeletal structure does not depend on the wild-type cellular geometry (Fig. 3C).

Next, we wondered whether CrvA and CrvB have distinct roles in assembling the periplasmic filament. Deleting crvA resulted in diffuse CrvB-GFP localization, indicating that CrvA is required for CrvB assembly (Fig. 4A). Deleting $c r v B$ also significantly disrupted CrvA-GFP, but in a qualitatively different manner. While most $\Delta c r v B$ cells still exhibited well-defined CrvAGFP structures, these cells typically exhibited multiple small CrvA-GFP puncta and only $\sim 6 \%$ had long CrvA-msfGFP filaments similar to those seen in wild type (Fig. 4A). We thus hypothesized that CrvA can form small structures on its own and that CrvB promotes the higherorder assembly of CrvA. To test this model, we placed $\operatorname{crvB}$ under arabinose-inducible control ( $\mathrm{P}_{\text {bad }}$ ) at an ectopic locus and observed the effect of $\operatorname{crvB}$ expression on CrvA-GFP assembly. Consistent with our hypothesis, $c r v B$ expression increased CrvA-GFP assembly (Figs. 4B and 4C) as well as cell curvature (Figs. 4B and 4D) in a dose-dependent manner. Higher levels of 
$c r v B$ expression also caused earlier onset of CrvA-GFP assembly (Fig. 4C), raising the question of whether the dynamics of CrvA-GFP assembly are important for the dynamics of $V$. cholerae curvature.

To assess the relationship between CrvA-GFP assembly and cell curvature dynamics, we simultaneously observed CrvA-GFP assembly and cell curvature in a wild-type background. Cell curvature decreased as CrvA-GFP filaments were lost (Fig. 4E), and in these straight cells, CrvA-GFP formed multiple small structures resembling those in $\triangle c r v B$. Our time course also showed that cell curvature only began to increase after the formation of long CrvA-GFP filaments (Fig. 4E). Taken together these results demonstrate that the CrvAB module forms a dynamic periskeletal structure, that CrvB promotes the formation of this higher-order structure, and that the timing of its assembly correlates to the dynamics of curvature.

\section{Discussion}

Together, our results demonstrate that both CrvA and CrvB have specialized roles in the assembly of a periskeletal structure that can impart cell shape complexity onto bacterial species separated by 2.5 billion years of evolution ${ }^{23}$. The ability of CrvAB to modulate cell shape independently of species-specific growth mechanisms could have bioengineering applications like improving the stability of synthetic probiotics, as cell curvature can enhance intestinal colonization ${ }^{14}$. The periskeletal nature of CrvAB allows this module to generate asymmetry similarly to cytoskeletal elements, but without requiring transmembrane interaction partners to influence periplasmic cell wall synthesis. Most cytoskeletal elements require ATP or GTP for dynamics. These energy sources are absent in the periplasm, but CrvAB demonstrate that 
periplasmic polymers can be dynamic nevertheless. As PG is chemically similar among bacteria

${ }^{24}$, a direct interaction with the cell wall may provide an explanation for the unprecedented range of species in which $\mathrm{CrvAB}$ are functional. Alternatively, CrvAB may produce asymmetry by directing the activity of PG enzymes that function outside of the core cell shape complexes. In a variety of species, determinants of complex shape have been identified ${ }^{3,25}$, but it remains to be seen if these systems depend on core shape machinery or if they will join CrvAB as autonomous cell shape modules. Furthermore, there are complex bacterial morphologies without characterized determinants. Perhaps these diverse forms are built not by co-option of core cell biology, but by simple cell shape modules like the one that makes $V$. cholerae curved.

\section{References and Notes:}

1 Young, K. D. The selective value of bacterial shape. Microbiol Mol Biol Rev 70, 660703, doi:10.1128/MMBR.00001-06 (2006).

2 Yang, D. C., Blair, K. M. \& Salama, N. R. Staying in Shape: the Impact of Cell Shape on Bacterial Survival in Diverse Environments. Microbiol Mol Biol Rev 80, 187-203, doi:10.1128/MMBR.00031-15 (2016).

3 Taylor, J. A., Sichel, S. R. \& Salama, N. R. Bent Bacteria: A Comparison of Cell Shape Mechanisms in Proteobacteria. Annu Rev Microbiol 73, 457-480, doi:10.1146/annurevmicro-020518-115919 (2019).

4 Schuech, R., Hoehfurtner, T., Smith, D. J. \& Humphries, S. Motile curved bacteria are Pareto-optimal. Proc Natl Acad Sci U S A 116, 14440-14447, doi:10.1073/pnas.1818997116 (2019).

5 Billini, M., Biboy, J., Kuhn, J., Vollmer, W. \& Thanbichler, M. A specialized MreBdependent cell wall biosynthetic complex mediates the formation of stalk-specific peptidoglycan in Caulobacter crescentus. PLoS Genet 15, e1007897, doi:10.1371/journal.pgen.1007897 (2019).

6 Charbon, G., Cabeen, M. T. \& Jacobs-Wagner, C. Bacterial intermediate filaments: in vivo assembly, organization, and dynamics of crescentin. Genes Dev 23, 1131-1144, doi:10.1101/gad.1795509 (2009).

7 Orr, R. G., Cheng, X., Vidali, L. \& Bezanilla, M. Orchestrating cell morphology from the inside out - using polarized cell expansion in plants as a model. Curr Opin Cell Biol 62, 46-53, doi:10.1016/j.ceb.2019.08.004 (2019).

8 Vogel, V. \& Sheetz, M. Local force and geometry sensing regulate cell functions. Nat Rev Mol Cell Biol 7, 265-275, doi:10.1038/nrm1890 (2006).

9 Typas, A., Banzhaf, M., Gross, C. A. \& Vollmer, W. From the regulation of peptidoglycan synthesis to bacterial growth and morphology. Nat Rev Microbiol 10, 123136, doi:10.1038/nrmicro2677 (2011). 
10 Ursell, T. S. et al. Rod-like bacterial shape is maintained by feedback between cell curvature and cytoskeletal localization. Proc Natl Acad Sci U S A 111, E1025-1034, doi:10.1073/pnas.1317174111 (2014).

11 Garner, E. C. et al. Coupled, circumferential motions of the cell wall synthesis machinery and MreB filaments in B. subtilis. Science 333, 222-225, doi:10.1126/science.1203285 (2011).

12 Yang, X. et al. GTPase activity-coupled treadmilling of the bacterial tubulin FtsZ organizes septal cell wall synthesis. Science 355, 744-747, doi:10.1126/science.aak9995 (2017).

13 Bisson-Filho, A. W. et al. Treadmilling by FtsZ filaments drives peptidoglycan synthesis and bacterial cell division. Science 355, 739-743, doi:10.1126/science.aak9973 (2017).

14 Bartlett, T. M. et al. A Periplasmic Polymer Curves Vibrio cholerae and Promotes Pathogenesis. Cell 168, 172-185 e115, doi:10.1016/j.cell.2016.12.019 (2017).

15 Farmer Iii, J. J., Michael Janda, J., Brenner, F. W., Cameron, D. N. \& Birkhead, K. M. Vibrio. Bergey's Manual of Systematics of Archaea and Bacteria, 1-79 (2015).

16 Cowles, K. N. \& Gitai, Z. Surface association and the MreB cytoskeleton regulate pilus production, localization and function in Pseudomonas aeruginosa. Mol Microbiol 76, 1411-1426, doi:10.1111/j.1365-2958.2010.07132.x (2010).

17 Srivastava, P., Demarre, G., Karpova, T. S., McNally, J. \& Chattoraj, D. K. Changes in nucleoid morphology and origin localization upon inhibition or alteration of the actin homolog, MreB, of Vibrio cholerae. J Bacteriol 189, 7450-7463, doi:10.1128/JB.0036207 (2007).

18 Cameron, T. A., Anderson-Furgeson, J., Zupan, J. R., Zik, J. J. \& Zambryski, P. C. Peptidoglycan synthesis machinery in Agrobacterium tumefaciens during unipolar growth and cell division. Mbio 5, e01219-01214, doi:10.1128/mBio.01219-14 (2014).

19 Howell, M. et al. Agrobacterium tumefaciens divisome proteins regulate the transition from polar growth to cell division. Mol Microbiol 111, 1074-1092, doi:10.1111/mmi.14212 (2019).

20 Ouzounov, N. et al. MreB Orientation Correlates with Cell Diameter in Escherichia coli. Biophys J 111, 1035-1043, doi:10.1016/j.bpj.2016.07.017 (2016).

21 Schierle, C. F. et al. The DsbA signal sequence directs efficient, cotranslational export of passenger proteins to the Escherichia coli periplasm via the signal recognition particle pathway. J Bacteriol 185, 5706-5713, doi:10.1128/jb.185.19.5706-5713.2003 (2003).

22 Weaver, A. I. et al. Genetic Determinants of Penicillin Tolerance in Vibrio cholerae. Antimicrob Agents Chemother 62, doi:10.1128/AAC.01326-18 (2018).

23 Battistuzzi, F. U., Feijao, A. \& Hedges, S. B. A genomic timescale of prokaryote evolution: insights into the origin of methanogenesis, phototrophy, and the colonization of land. BMC Evol Biol 4, 44, doi:10.1186/1471-2148-4-44 (2004).

24 Taguchi, A., Kahne, D. \& Walker, S. Chemical tools to characterize peptidoglycan synthases. Curr Opin Chem Biol 53, 44-50, doi:10.1016/j.cbpa.2019.07.009 (2019). Caccamo, P. D. \& Brun, Y. V. The Molecular Basis of Noncanonical Bacterial Morphology. Trends Microbiol, doi:10.1016/j.tim.2017.09.012 (2017).

26 Sawabe, T. et al. Updating the Vibrio clades defined by multilocus sequence phylogeny: proposal of eight new clades, and the description of Vibrio tritonius sp. nov. Front Microbiol 4, 414, doi:10.3389/fmicb.2013.00414 (2013). 
27 Skorupski, K. \& Taylor, R. K. Positive selection vectors for allelic exchange. Gene 169, 47-52 (1996).

28 Edwards, R. A., Keller, L. H. \& Schifferli, D. M. Improved allelic exchange vectors and their use to analyze 987P fimbria gene expression. Gene 207, 149-157, doi:10.1016/s0378-1119(97)00619-7 (1998).

29 Bina, X. R., Wong, E. A., Bina, T. F. \& Bina, J. E. Construction of a tetracycline inducible expression vector and characterization of its use in Vibrio cholerae. Plasmid 76, 87-94, doi:10.1016/j.plasmid.2014.10.004 (2014).

30 Guzman, L. M., Belin, D., Carson, M. J. \& Beckwith, J. Tight regulation, modulation, and high-level expression by vectors containing the arabinose PBAD promoter. $J$ Bacteriol 177, 4121-4130 (1995).

31 Katoh, K. \& Standley, D. M. MAFFT multiple sequence alignment software version 7: improvements in performance and usability. Mol Biol Evol 30, 772-780, doi:10.1093/molbev/mst010 (2013).

32 Henikoff, S. \& Henikoff, J. G. Amino acid substitution matrices from protein blocks. Proc Natl Acad Sci U S A 89, 10915-10919, doi:10.1073/pnas.89.22.10915 (1992).

33 Criscuolo, A. \& Gribaldo, S. BMGE (Block Mapping and Gathering with Entropy): a new software for selection of phylogenetic informative regions from multiple sequence alignments. BMC Evol Biol 10, 210, doi:10.1186/1471-2148-10-210 (2010).

34 Guindon, S. et al. New algorithms and methods to estimate maximum-likelihood phylogenies: assessing the performance of PhyML 3.0. Syst Biol 59, 307-321, doi:10.1093/sysbio/syq010 (2010).

35 Capra, J. A. \& Singh, M. Predicting functionally important residues from sequence conservation. Bioinformatics 23, 1875-1882, doi:10.1093/bioinformatics/btm270 (2007).

36 Sievers, F. et al. Fast, scalable generation of high-quality protein multiple sequence alignments using Clustal Omega. Mol Syst Biol 7, 539, doi:10.1038/msb.2011.75 (2011).

37 Thelin, K. H. \& Taylor, R. K. Toxin-coregulated pilus, but not mannose-sensitive hemagglutinin, is required for colonization by Vibrio cholerae O1 El Tor biotype and O139 strains. Infect Immun 64, 2853-2856 (1996).

38 Simon, R., Quandt, J. \& Klipp, W. New derivatives of transposon Tn5 suitable for mobilization of replicons, generation of operon fusions and induction of genes in gramnegative bacteria. Gene 80, 161-169, doi:10.1016/0378-1119(89)90262-x (1989).

39 Blattner, F. R. et al. The complete genome sequence of Escherichia coli K-12. Science 277, 1453-1462, doi:10.1126/science.277.5331.1453 (1997).

40 Rahme, L. G. et al. Common virulence factors for bacterial pathogenicity in plants and animals. Science 268, 1899-1902, doi:10.1126/science.7604262 (1995).

41 Evinger, M. \& Agabian, N. Envelope-associated nucleoid from Caulobacter crescentus stalked and swarmer cells. J Bacteriol 132, 294-301 (1977).

42 Gitai, Z., Dye, N. \& Shapiro, L. An actin-like gene can determine cell polarity in bacteria. Proc Natl Acad Sci U S A 101, 8643-8648, doi:10.1073/pnas.0402638101 (2004).

43 Watson, B., Currier, T. C., Gordon, M. P., Chilton, M. D. \& Nester, E. W. Plasmid required for virulence of Agrobacterium tumefaciens. J Bacteriol 123, 255-264 (1975).

44 Boettcher, K. J. \& Ruby, E. G. Depressed light emission by symbiotic Vibrio fischeri of the sepiolid squid Euprymna scolopes. J Bacteriol 172, 3701-3706, doi:10.1128/jb.172.7.3701-3706.1990 (1990). 
45 Dunn, A. K., Millikan, D. S., Adin, D. M., Bose, J. L. \& Stabb, E. V. New rfp- and pES213-derived tools for analyzing symbiotic Vibrio fischeri reveal patterns of infection and lux expression in situ. Appl Environ Microbiol 72, 802-810, doi:10.1128/AEM.72.1.802-810.2006 (2006).

46 Schweizer, H. P. Escherichia-Pseudomonas shuttle vectors derived from pUC18/19. Gene 97, 109-121, doi:10.1016/0378-1119(91)90016-5 (1991).

47 Thanbichler, M., Iniesta, A. A. \& Shapiro, L. A comprehensive set of plasmids for vanillate- and xylose-inducible gene expression in Caulobacter crescentus. Nucleic Acids Res 35, e137, doi:10.1093/nar/gkm818 (2007).

Acknowledgments: We thank members of the Gitai and Shaevitz labs for helpful discussions,

the Bina and Bassler labs for reagents, Joe Sanfilippo, Matthias Koch, Courtney Ellison, Josh

Shaevitz, and Tom Silhavy for helpful feedback on the manuscript. Funding: This work was

supported by a grant (1DP1AI124669) from the National Institutes of Health (to Z.G.) and

Graduate Research Fellowships from the National Science Foundation (to N.R.M. and T.M.B.)

Author contributions: All experiments were performed by N.R.M., E.B., and T.M.B. with

design input from Z.G. Data and sequence analysis were performed by N.R.M. and B.P.B. The

manuscript was written by Z.G. and N.R.M. with input from the other authors. Competing

interests: Authors declare no competing interests. Data and materials availability: All data is available in the main text or the supplementary materials.

\section{Supplementary Materials:}

Figures S1-S5

Tables S1-S2

Movies S1-S2

References (27-47) 


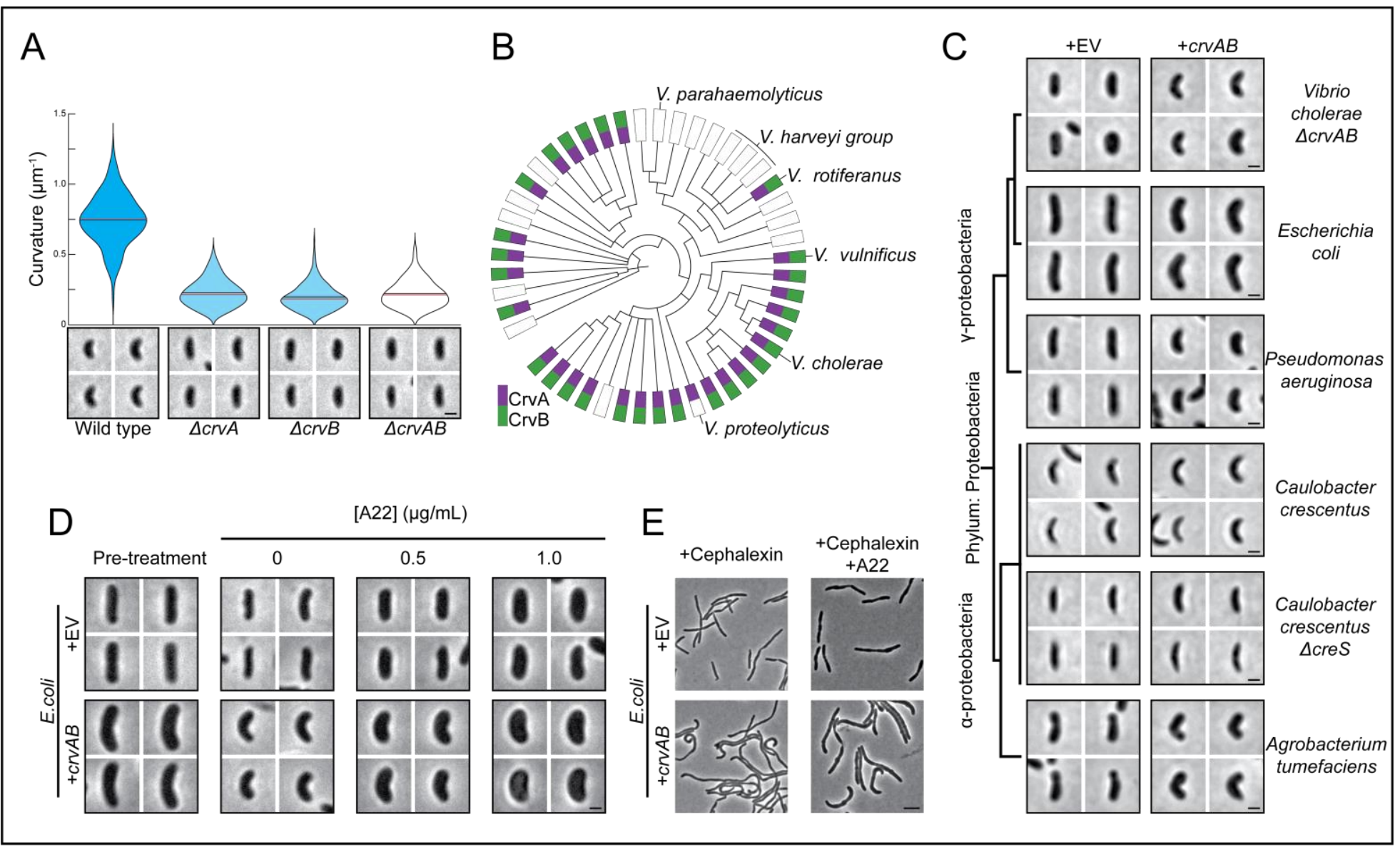

Fig. 1. CrvA and CrvB curve bacterial cells. (A) Single-cell curvature measurements using

Morphometrics ${ }^{10}$ from indicated V. cholerae strains. Bars represent mean (black) and median (red). (B) Presence of CrvA and CrvB homologs across Vibrio species. (C-E) Heterologous expression of $\operatorname{crv} A$ and $\operatorname{crv} B(+\operatorname{crv} A B)$ or empty vector (+EV). (C) Brackets indicate phylogenetic relationships between species. (D) E. coli before (Pre-treatment) and after A22 treatment (E) E. coli after treatment with cephalexin and A22. (A,C,D) Images represent $95^{\text {th }}$ percentile of curvature in respective populations. Scale bars are $1 \mu \mathrm{m}$. (B) Clade structure drawn according to ${ }^{26}$. (C) Median curvature of + crvAB is significantly higher than $+E V$ in each species $\left(\mathrm{p} \leq 3.74 \times 10^{-5}\right)$. (D) Median curvature of $+\mathrm{crvAB}$ is significantly higher than $+\mathrm{EV}$ and Pretreatment at each A22 concentration $\left(\mathrm{p} \leq 2.02 \times 10^{-9}\right)$. Wilcoxon rank sum tests used for all statistics; $n=300$ (See FigS2 for quantified populations). Scale bars represent $1 \mu \mathrm{m}$ in (A,C,D) and $5 \mu \mathrm{m}$ in (E). 


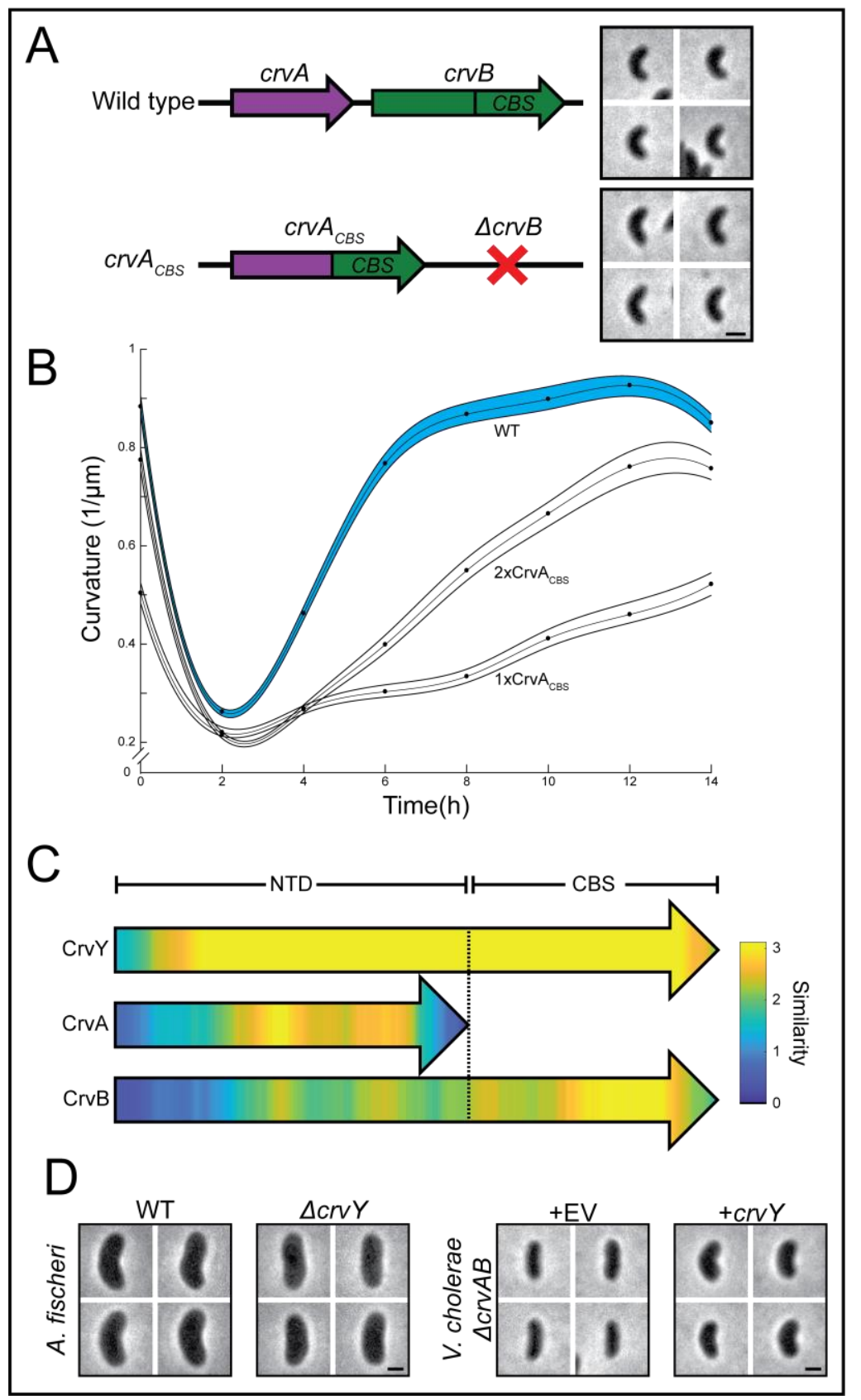

Fig. 2. CrvA and CrvB are functionally and structurally specialized. (A) Schematic of the $\operatorname{crv} A B$ locus and representative images of wild type and cells expressing the $\operatorname{crv} A_{C B S}$ chimera. (B) Dynamics of mean curvature \pm 95\% confidence intervals (bootstrapped) in wild type (WT) and strains expressing one ( $\left.1 \mathrm{xCrv}_{\mathrm{CBS}}\right)$ or two $\left(2 \mathrm{xCrv}_{\mathrm{CBS}}\right)$ copies of $c r v A_{C B S .}$. (C) Pairwise sequence alignment of all sequenced CrvA, CrvB, and CrvY homologs to CrvY. Sequence alignment and local similarity using the BLOSUM80 scoring matrix. (D) Deletion of $c r v Y$ in $A$. fischeri and expression of $c r v Y_{A}$. fischeri in $V$. cholerae $\triangle$ crv $A B$. (A,D) Images represent $95^{\text {th }}$ percentile of curvature in respective populations. Scale bars represent $1 \mu \mathrm{m}$. See FigS4 for population measurements. 


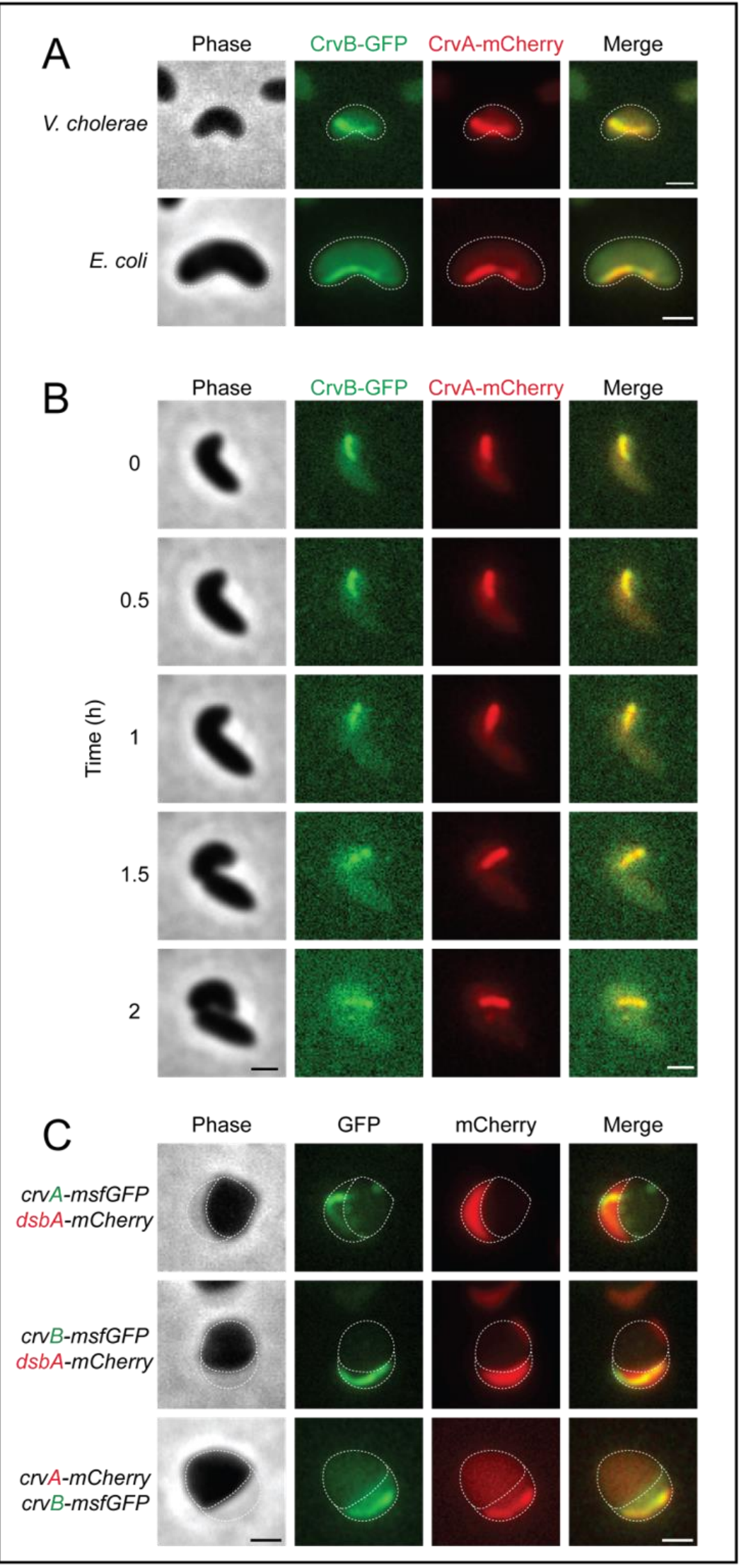

Fig. 3. CrvA and CrvB colocalize in a

periplasmic filament. (A) Curved $V$.

cholerae and E. coli cells expressing

CrvB-GFP and CrvA-mCherry fusions.

(B) Time lapse images of CrvB-GFP

and CrvA-mCherry structures in

growing $V$. cholerae. See Movie S1 for

full time lapse. (C) Fluorescent CrvA

and CrvB filaments with periplasmic

DsbA-mCherry in $V$. cholerae after

moenomycin treatment. $(\mathrm{A}, \mathrm{C})$ Dotted

lines represent outline of cell body and

periplasm. Scale bars represent $1 \mu \mathrm{m}$. 


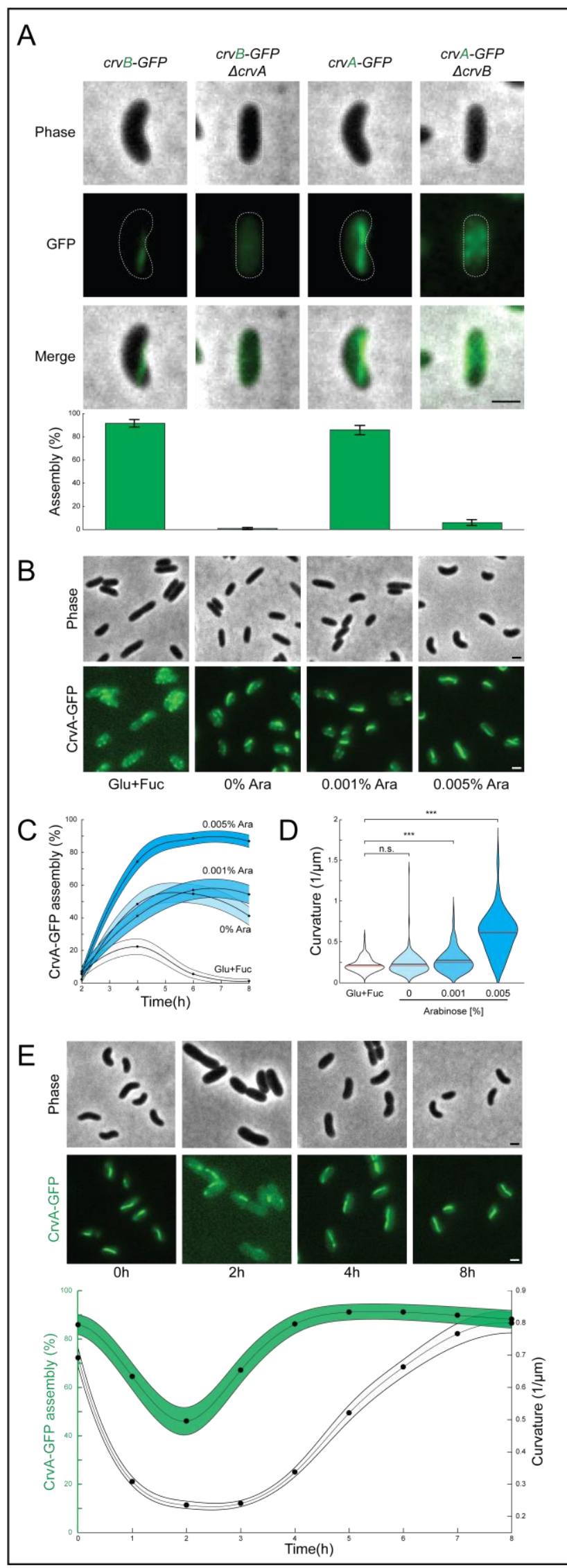

Fig. 4. CrvA and CrvB synergize to promote

filament assembly in $V$. cholerae. (A)

Representative cells (top) and quantified CrvA-GFP and CrvB-GFP filament assembly (bottom, error bars indicate $\pm 95 \%$ confidence intervals) in deletion mutants. (B) Representative fields after repression (Glu+Fuc) and induction (Ara) of $P_{b a d^{-}} c r v B$. (C) Dynamics of CrvA-GFP assembly from (B) $\pm 95 \%$ confidence intervals. (D) End point curvature measurements from (B). (E) Dynamics of mean curvature and CrvA-GFP filament assembly $\pm 95 \%$ confidence intervals. (A-E) Filament assembly is the percentage of cells with fluorescent filaments similar to those in the wild type background. $n=300$;

Wilcoxon rank-sum test; n.s. $=\mathrm{p}>0.4, * * *=\mathrm{p}<0.0001$. $95 \%$ confidence intervals on filament assembly and curvature determined by Agresti-Coull method and bootstrapping, respectively. Scale bars represent $1 \mu \mathrm{m}$. 


\section{Materials and Methods}

\section{$\underline{\text { Bacterial strains and growth conditions }}$}

Bacterial strains and plasmids used are listed in Table S1. Vibrio cholerae, Escherichia coli, Pseudomonas aeruginosa were cultured in LB medium (10g/L NaCl (Sigma), 10g/L tryptone (Bacto), 5g/L yeast extract (Bacto)) at $37^{\circ} \mathrm{C}$ with aeration. Agrobacterium tumefaciens was cultured in $\mathrm{LB}$ medium at $30^{\circ} \mathrm{C}$ with aeration. Alivibrio fischeri was cultured either on LB agar (Bacto) plates or in $\mathrm{LM}$ liquid medium $\left(20 \mathrm{~g} / \mathrm{L} \mathrm{NaCl}, 10 \mathrm{~g} / \mathrm{L}\right.$ tryptone, $5 \mathrm{~g} / \mathrm{L}$ yeast extract) at $30^{\circ} \mathrm{C}$ with aeration. Caulobacter crescentus was grown in PYE medium (2g/L peptone (Bacto), 1g/L Yeast extract, $1 \mathrm{mM} \mathrm{MgSO}_{4}(\mathrm{Sigma}), 0.5 \mathrm{mM} \mathrm{CaCl}_{2}$ (Sigma)) at $30^{\circ} \mathrm{C}$ with aeration. Cultures in Fig 3 and Fig 4B were grown in 2mL culture volumes, while all other experiments were conducted in $5 \mathrm{~mL}$ culture volumes. Chromosomal mutations in V. cholerae and A. fischeri were made using suicide vectors pKAS32(Skorupski and Taylor, 1996) and pRE112(Edwards et al., 1998), respectively. Plasmids were introduced by conjugal transfer from E. coli S17 into $V$.

cholerae, C. crescentus, A. tumefaciens, and A. fischeri. Plasmids were introduced by electroporation into $P$. aeruginosa and E. coli MG1655. Antibiotics were used in the following

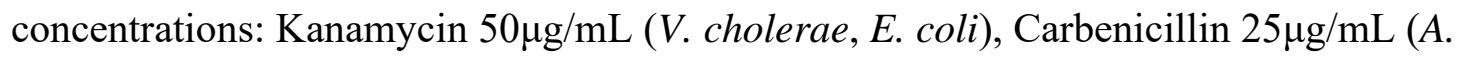
tumefaciens), Carbenicillin $200 \mu \mathrm{g} / \mathrm{mL}$ (P.aeruginosa), Chloramphenicol 1 $\mu \mathrm{g} / \mathrm{mL}(C$. crescentus).

\section{$\underline{\text { Microscopy }}$}

Images were obtained using a Hamamatsu ORCA-Flash4.0 Digital CMOS camera on either a Nikon 90i microscope with a Nikon 100x Plan Apo NA=1.40 oil immersion objective lens or a Nikon Ti-E microscope with a Nikon 100x Plan Apo NA=1.45 oil immersion objective lens. For 
fluorescence, GFP-L and Cy5 HYQ filter cubes (90i) or GFP-HQ and mCherry filter cubes (TiE) were used to visualize msfGFP and mCherry fluorescent fusions, respectively. Excelitas X-

Cite 120 LEDBoost was used for illumination on both microscopes. All hardware was controlled by NIS Elements (version 4.60.00). Suspensions of $A$. fischeri cells were spotted on 1-2\% (weight/volume) agarose(Invitrogen) pads of LM for imaging. For all other species, 1-2\% agarose pads of M9 Salts (47.8mM Na $2 \mathrm{HPO}_{4}(\mathrm{Sigma}), 22 \mathrm{mM} \mathrm{KH} \mathrm{KO}_{4}(\mathrm{Sigma}), 8.6 \mathrm{mM} \mathrm{NaCl}$, 18.6mM NH $\mathrm{m}_{4} \mathrm{Cl}(\mathrm{Sigma})$ ) were used. For growth on pads, exponentially growing $V$. cholerae were spotted on 1-2\% agarose pads of M9 supplemented with $0.5 \%$ glucose (Sigma) and incubated at $25^{\circ} \mathrm{C}$ throughout the experiment. All geometric measurements were performed on phase-contrast images at 100X magnification. For merged images, problems with filter alignment were manually corrected by adjusting contrast such that the cell body but not features within the cell were clearly visible. The image plane was then shifted so that outline of this fluorescence was aligned to the phase-contrast image or other fluorescent channel.

\section{$\underline{\text { Measurement of cellular geometry }}$}

Phase images were analyzed using the MATLAB script Morphometrics(Ursell et al., 2014) to segment cell contours and fit them with centerlines. Contours touching adjacent cells or having visible cleavage furrows were excluded from analysis to avoid overestimation of curvature due to the orientation of neighboring cells or recently divided cells. Curvature is root mean squared curvature of the centerline in units of inverse microns $\left(\mu \mathrm{m}^{-1}\right)$. Width is mean width of the contour along the entire centerline in microns $(\mu \mathrm{m})$. Population measurements are from pooled measurements of 100 individual cells from three biological replicates performed on different days (300 cells per measurement). To account for differences in the number of cell contours in 
each of the three replicates, 100 cells were randomly selected from each replicate before pooling. Violin plots represent one such randomized population. Populations that were also measured for CrvA/CrvB-msfGFP filament assembly were not randomized because only the first 100 valid contours from each replicate were analyzed; therefore, all three replicates had an equal number of contours. For statistical tests of populations, the Wilcoxon two-sided rank sum test was used. For tests on randomized populations, the random sampling and statistical testing was repeated one thousand times and the mean p-value was reported to minimize the effect of any one subsample. Representative cells shown in Figs 1A,C,D, Fig 4D, Figs S1-S5 are representative of the $3^{\text {rd }}$ quartile of length in their respective populations to facilitate visual comparison of cells with similar lengths. Unless otherwise indicated, these cells represent the $95^{\text {th }}$ quantile of curvature in their respective populations.

\section{Measurement of CrvA/CrvB-msfGFP filament assembly}

Phase/GFP image stacks were split into their constituent channels and the phase channel was used to obtain cell contours using Morphometrics. After excluding touching and dividing cells as described above, the first 100 contours from each sample were superimposed on the corresponding GFP image. The GFP signal of each contour was manually inspected and categorized as "assembled" if most of the fluorescence signal formed a single, linear structure at least half the length of the cell. This was repeated on contours from three biological replicates performed on different days and pooled for population measurements (300 cells total). The Agresti-Coull method was used to estimate the $95 \%$ confidence intervals of this measurement and the center of this interval is reported as the proportion of the population that has assembled 
GFP structures. This proportion is referred to as the "assembly" of the population and is expressed as a percentage.

Complementation and heterologous expression of $\operatorname{crv} A, \operatorname{crvB}$, and $\operatorname{crv} Y$

For $\operatorname{crvA}$ or $\operatorname{crvB}$ expression, plasmid-borne alleles were placed under the control of a tetracycline-inducible promoter(Bina et al., 2014) and the following ribosome-binding site:

AGGAGCTAAGGAAGCTAAA. When expressed together, both open reading frames are under control of the same promoter separated by the same ribosome-binding site. The same expression fragment was cloned into different plasmid backbones when required for heterologous expression. Anhydrotetracycline was added at the beginning of subculture in the following concentrations with a final culture volume of $5 \mathrm{~mL}: 0 \mathrm{ng} / \mathrm{mL}$ for $V$. cholerae, E. coli, and $P$. aeruginosa; $2 \mathrm{ng} / \mathrm{mL}$ for A. tumefaciens; $5 \mathrm{ng} / \mathrm{mL}$ for $C$. crescentus. For $V$. cholerae, E. coli, and P. aeruginosa saturated overnight cultures were diluted 1:1000 into LB with appropriate antibiotics and grown for $6 \mathrm{~h}$ before imaging. For $C$. crescentus saturated overnight cultures were diluted 1:100 into PYE with appropriate antibiotics and grown for $7 \mathrm{~h}$ before imaging. For $A$. tumefaciens saturated overnight cultures were diluted 1:20 into LB with appropriate antibiotics and grown for $6 \mathrm{~h}$ before imaging. For $c r v Y$ expression in $V$. cholerae $\triangle \operatorname{crvAB}$, a plasmid-borne $\operatorname{crv} Y$ allele from $A$. fischeri was placed under the control of an arabinose-inducible promoter(Guzman et al., 1995) and the ribosome-binding site above. 0.1\% L-Arabinose (Sigma) was added to LB with Kanamycin at the beginning of subculture with a final culture volume of 5mL. Saturated overnight cultures were diluted to an optical density $\left(\mathrm{OD}_{600}\right)$ of 0.001 and grown for $4 \mathrm{~h}$ before imaging.

Sublethal treatment with A22, Cephalexin, and Moenomycin 
For moenomycin treatment saturated overnight LB cultures were diluted 1:1000 into fresh LB supplemented with $500 \mathrm{ng} / \mathrm{mL}$ moenomycin and incubated at $37^{\circ} \mathrm{C}$ for $5-6 \mathrm{~h}$ before imaging. Representative cells were chosen such that their orientation relative to the imaging plane allowed visual resolution of the periplasmic and cytoplasmic spaces. For A22 and cephalexin treatment, saturated overnight LB cultures were diluted 1:1000 into fresh LB and grown for $4 \mathrm{~h}$ after which pre-treatment samples were imaged. At 4h A22 and cephalexin were added in indicated concentrations. For A22 treatment cells were grown for an additional 4 h before imaging. For Cephalexin treatment, and simultaneous A22+cephalexin treatment cells were imaged $2 \mathrm{~h}$ after drug addition.

\section{Titration of $\operatorname{crvB}$ expression in $V$. cholerae}

$c r v B$ was deleted in a strain background expressing $c r v A-m s f G F P$ from the native locus producing NM149. Into this background, a wild-type $\operatorname{crvB}$ was introduced at an ectopic locus on the opposite chromosome ( VC1378) under the control of an arabinose-inducible promoter (Guzman et al., 1995) producing NM565. Saturated vernight cultures of NM565 were grown under repressing conditions (0.05\% D-Fucose(Acros), $0.5 \%$ D-Glucose) in LB and back-diluted to an $\mathrm{OD}_{600}$ of 0.001 into repressing conditions or into LB supplemented with indicated concentrations of L-Arabinose. Images were taken starting $2 \mathrm{~h}$ after back-dilution.

\section{Phylogenetic analysis}

A multiple alignment of the unique sequences of crv proteins (all found within the Vibrionales clade) and an outgroup of the closest homologs found after excluding the Vibrionales was made using MAFFT(Katoh and Standley, 2013) with blosum80(Henikoff and Henikoff, 1992) as the 
scoring matrix. Following this multiple alignment, a phylogenetic tree was constructed using BMGE/FastTree(Criscuolo and Gribaldo, 2010; Guindon et al., 2010). To compare the local sequence similarity between the CrvA, CrvB, and CrvY, we divided the sequences into four clades $(\mathrm{c}=\{$ outgroup, crvA, crvB, crvY $\})$ and calculated a local, windowed alignment score(Capra and Singh, 2007) compared to the CrvY clade using the blosum80 scoring matrix.

\author{
Score(iStrain, jReference, kPosition) \\ = blosum80(sequence(iStrain, kPosition), sequence(jReference, $k$ Position) \\ WindowedScore(iStrain,jReference, kPosition) \\ $=\frac{\sum_{\text {delta }=+ \text { windowSize }}^{\text {delta }} \text { Score }(\text { iStrain }, j \text { Reference }, k \text { Position }- \text { delta })}{\mid 2 * \text { window size }+1 \mid}$
}

AveragedWindowedScore(kPosition)

$$
=\frac{\sum_{\text {strains }} \sum_{\text {references }} \text { WindowedScore }(\text { iStrain }, j \text { Reference, } k \text { Position })}{\text { nStrains nReferences }}
$$

